\title{
H.264/AVC Video Encoder Realization and Acceleration on TI DM642 $\mathrm{DSP}^{\star}$
}

\author{
Daw-Tung Lin and Chung-Yu Yang \\ Department of Computer Science and Information Engineering \\ National Taipei University \\ 151, University Rd., San-Shia, Taipei, 237 Taiwan \\ dalton@mail.ntpu.edu.tw
}

\begin{abstract}
This work develops and optimizes H.264/AVC video encoder on the TM320DM642 DSP platform. In order to transplant x264 source program onto the DSP and to accelerate the coding speed, a series of optimization methods have been proposed in this paper, including 2-D fast mode decision, sub-pixel optimization for motion estimation, and weighted matrix quantization. Furthermore, based on the architectural features of TM320DM642, various system level optimization techniques have been utilized. This paper focuses on the reduction of algorithm complexity. Experimental results reveal that the optimized H.264 video encoder retains satisfactory quality with very low degradation. The implemented codec can achieve the coding speed of 22.6fps and more than 40fps for VGA $(640 \times 480)$ and CIF $(352 \times 288)$ resolution, respectively. The proposed H.264 codec can be employed in many real-time applications.
\end{abstract}

Keywords: H.264/AVC encoder, TM320DM642 DSP, mode decision, motion estimation, quantization, optimization.

\section{Introduction}

H.264/AVC video coding technology provides better bit rate saving and high flexibility of use in a broad variety of domains video compression standards. However, the computational complexity of H.264 is much higher. It is important to optimize and realize an H.264 encoder for real time applications. Special purpose H.264 IC and embedded system are common solutions. Besides, the embedded software approach possesses the advantage of flexibility in updating and adding new functions. The embedded system has more potential.

In the recent years, a large amount of works have been focused on the development and optimization of H.264 algorithms. However, rare literatures disclosed the impact of implementing H.264 on the embedded system. We believe that creating H.264 video encoding/decoding application with good performance will

\footnotetext{
* This work was supported in part by the National Science Council, Taiwan, R.O.C. grants NSC96-2622-E-305-001-CC3 and Ministry of Economics grant: Construction of Vision-Based Intelligent Environment (II).
}

T. Wada, F. Huang, and S. Lin (Eds.): PSIVT 2009, LNCS 5414, pp. 910-920, 2009.

(C) Springer-Verlag Berlin Heidelberg 2009 
amplify the value of H.264. Recently, the improvement and implementation of H.264 codec on embedded system is getting more and more important. K. Goto et al. ported H.264 algorithm on a low-power DSP which achieves 15fps for QVGA $(320 \times 240)$ resolution [1]. T.-C. Chen et al. proposed a low power integer motion estimation algorithm and implemented it on a VLSI architecture with good performance [2]. The encoding speed achieves 30 fps for CIF $(352 \times 288)$ resolution. L. Zhuo and Z. Li also optimized the motion estimation algorithm and implemented on DM642 DSP platform with encoding speed of 24fps for CIF format video chips 34 . Z. Wei adopted advance assembly instruction and re-wrote kernel function and encoded the QCIF video in real-time speed [5]. Experiments of the limited storage resources on chip was also studied in detail to accelerate encoding speed. H.-J. Wang et al. and I. Werda et al. employed memory space allocation optimization to speed up the system which were realized on TMS320DM642 and TMS320C6416 DSP, respectively [6 7].

The remainder of this paper is organized as follows. Section2 gives an overview of the H.264 video encoder and the TI DM642 DSP. Section 3 then presents the proposed algorithm optimization approaches and the implementation. Section 4 analyzes the experimental results, and compares them with those of other encoding schemes implemented on DM642 DSP. Conclusions are finally drawn in Section 5 .

\section{Overview of H.264 and DM642 DSP}

H.264/AVC is an advanced video compression standard. It is also known as MPEG-4 Part 10. The main objective of the H.264/AVC is to create a standard capable of providing good video quality at substantially lower bit rates than the previous standards. H.264 has some features including low bit-rate, high quality, wide application, and robust video transmission (error resilient). In order to achieve the above features, H.264 employs many innovative techniques such as intra prediction, variable block size for inter coding, motion estimation with multiple reference frames, quarter pixel interpolation, $4 \times 4$ integer DCT, in-loop de-blocking filter, and advanced entropy coding (CAVLC and CABAC). By taking the advantages of these techniques, H.264 achieves a better performance than previous video coding standards like MPEG2 and MPEG4. Although these techniques improve the coding performance, they increase the complexity of the codec at the same time. Some literatures show that the complexity of H.264 is about 5 to 8 times that of H.263 8 . Therefore, it is very difficult to implement the H.264 codec in real time on the embedded system. How to optimize and implement H.264 codec is a very important research issue.

Texas Instruments TMS320DM642 digital signal processor is a general purpose processor with video in/out peripheral [9]. The clock rate of DM642 is $720 \mathrm{MHz}$. DM642 is one of the C6000 series with very long instruction word (VLIW) architecture which is able to issue a fixed number of instructions in parallel. In additional to the capability of high speed com-2 computation $(720 \mathrm{MHz}$, 
5760MIPS) and SIMD, the DM642 process can execute eight 32 bits instructions in each clock cycle. In other words, it is able to fetch a 256 bits long instruction from program memory in each clock cycle. There are two register banks in DM642 CPU core, four ALU units on each side, totally eight ALU units can execute instructions simultaneously. There are $16 \mathrm{k}$ bytes L1 data cache, $16 \mathrm{k}$ bytes L1 code cache, $256 \mathrm{k}$ bytes programmable L2 SRAM. There is a DMA controller on chip for data movement. Its EMDA offers 2GB per second I/O bandwidth in 64 independent channels [9].

\section{The Proposed Optimization Approach}

The objective of this work is to implement and optimize H.264 video encoding system on TI DM642EVM board based on x264 open source library. The x264 is one of the major open source encoder developed in 2004 licensed under the GPL [10. It performs the best in term of time complexity compared with most of the H.264-based algorithms. The coding speed of x264 is about 40-50 times faster than that of reference encoder JM (Joint Model) based on our simulations with video clips Foreman, Football, Akiyo and Water fall. As the major open-source H.264 encoder, x264 plays the role of a near-complete monopoly of H.264 encoder in the industry and has been used by many major corporations including Google Video, MobileASL, Speed Demos Archive, and TASvideos.

Before doing optimization, we analyze the complexity of each x264 function including the the kernel function clock cycle, execution time and code size. Figure 1 shows the run time percentage of each function. Apparently, motion estimation (ME) and macroblock size mode decision are the most time consuming functions. In order to transplant x264 source program onto the DSP and to accelerate the coding speed, a series of optimization methods have been proposed including 2-D fast mode decision, sub-pixel optimization for motion estimation, and weighted matrix quantization. For the other operations such as x264_clip, abs, sad, satd, etc., we rewrote these functions with assembly code and rearrange the instruction utilizing the features of hardware architecture.

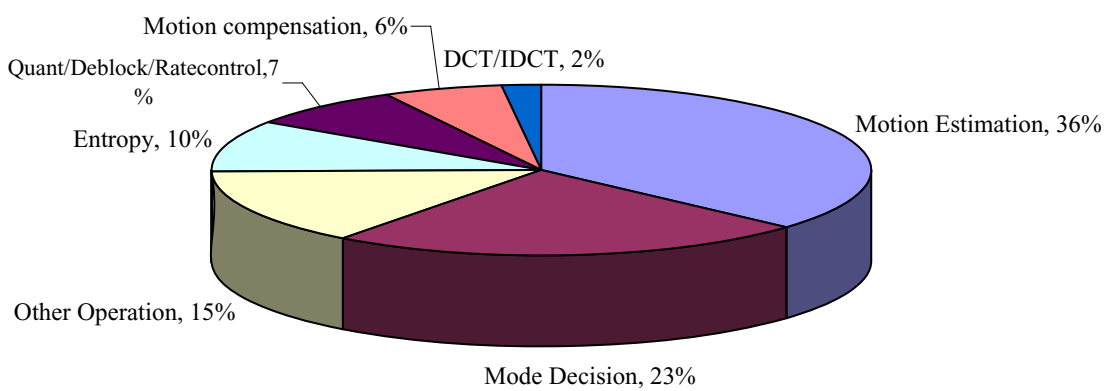

Fig. 1. Runtime percentage analysis of x264 kernel functions 


\subsection{Macroblock Coding 2-D Fast Mode Decision}

According to the analysis of the H.264 block modes distribution of many video sequences, the skip mode and $16 \times 16$ mode hold more than $50 \%$ of the block modes. When the macroblock is resided in the background or in the smooth region, the $16 \times 16$ or skip mode will be chosen, and the same mode will be clustered together. Chang et al. proposed a fast mode decision for P-frames in H.264 using the average rate-distortion (RD) cost threshold for the decision of early skip [11. We utilized this early skip method and further proposed a new mode decision algorithm which adapts to the hardware structure feature of DM642. The proposed 2-D fast mode decision is based on the mode status in time domain and spatial domain, taking advantage of those block information to reduce the complexity of mode decision. The detail analysis is referred to [11. We define three statuses of block mode as shown in Fig. 2. Gray block in Fig. 2 represents the current frame block to be decided. $A_{n}, B_{n}$ and $C_{n}$ denote the neighborhood block mode of the current frame. $D_{n-1}$ and $E_{n-1}$ are the block modes of the lower and right blocks, respectively, in previous frame. We further set $T_{1}$ as an integer threshold used to decided skip mode. Moment $T_{2}$ is firstly defined as the average R-D cost of $A_{n}, B_{n}$ and $C_{n}$. When more than two block modes of $A_{n}, B_{n}$ and $C_{n}$ are the same, $\operatorname{Cost}\left(M_{o d e}\right)$ will be defined as the R-D cost of the current block using this mode. Otherwise, the moment $T_{2}$ is set as the average R-D cost of $D_{n-1}$ and $E_{n-1}$. When the block modes of $D_{n-1}$ and $E_{n-1}$ are the same, $\operatorname{Cost}\left(\operatorname{Mode}_{n-1}\right)$ is define as the R-D cost of the current block using this mode. Then, the mode decision is based on the parameters of $T_{1}, T_{2}, \operatorname{Cost}\left(\operatorname{Mode}_{n}\right)$ and $\operatorname{Cost}\left(\right.$ Mode $\left._{n-1}\right)$. The main procedure of the proposed method is described as follows.

Step1: Check the skip mode, if R-D cost is small than $T_{1}$, choose skip mode and go to Step6.

Step2: If the MB is in the first row or first column, test all mode and select the best one, go to Step6.

Step3: Set $T_{2}$ equals to the average R-D cost of $A_{n}, B_{n}$ and $C_{n}$. Check MB $A_{n}, B_{n}$ and $C_{n}$, if more than two MB types are the same and $\operatorname{Cost}\left(\right.$ Mode $\left._{n}\right)<$ $T_{2}$, then use this mode, go to Step6.

Step4: Set $T_{2}$ equals to the average R-D cost of $D_{n-1}$ and $E_{n-1}$. Check MB $D_{n-1}$ and $E_{n-1}$, if the MB types are the same and $\operatorname{Cost}\left(\right.$ Mode $\left._{n-1}\right)<T_{2}$, then use this mode, go to Step6.

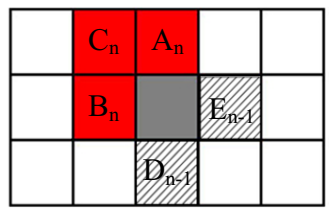

current frame block mode

decision block

previous frame block mode

Fig. 2. Three types of block modes 
Step5: Check the remaining modes.

Step6: Stop.

\subsection{Sub-pixel Motion Estimation Optimization}

One of the important motion estimation optimization issues is sub-pixel motion estimation. Sub-pixel motion estimation usually finds a better position than full-pixel motion estimation and therefore leads to a smaller SAD and bit rate. For sub-pixel motion estimation, half-pixel search is frequently used in H.263, MPEG-1, MPEG-2 and MPEG-4. Quarter-pixel search is adopted in MPEG-4 and H.264/AVC to achieve more accurate motion description and higher compression efficiency. In our system, sub-pixel ME needs sub-pixel interpolation for the whole search area before performing diamond search. However, it costs a lot of computation complexity. After finding the best position in full-pixel precision, it searches only eight neighboring half-pixel positions (denoted as square block in Fig. [3). When the motion vector moves from position "1" to position "3" (shown in Fig. (3) , the integer search starts from point "1", then the half-pixel search will find point "2". Finally point "3" will be found by quarter-pixel search. Due to high complexity of the sub-pixel search algorithm, we use integer-pixel search to engage the first sub-pixel search, then use quarter-pixel search to finish the subpixel motion estimation. This search method improves the sub-pixel searching speed avoiding complicated computation and is useful for our DSP platform.

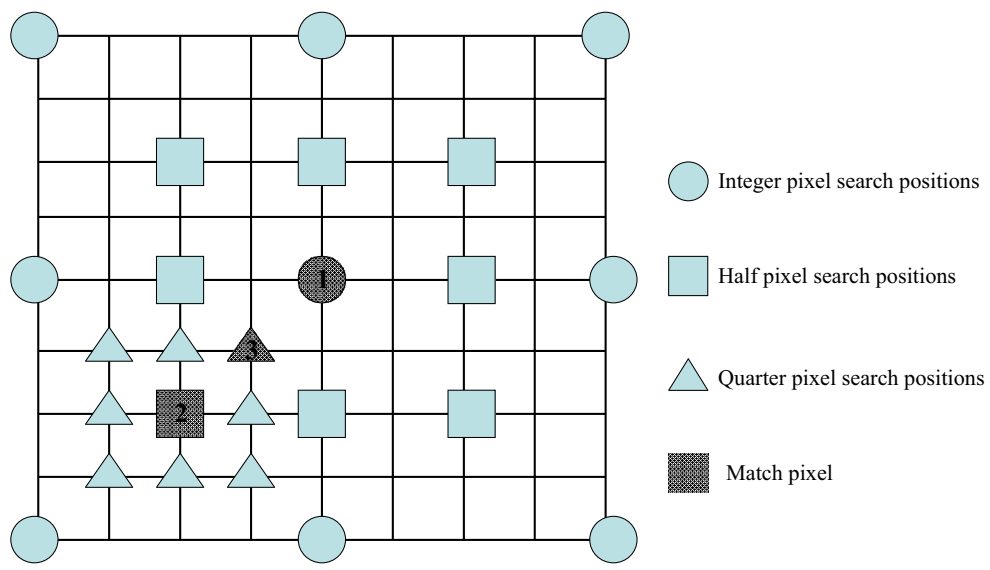

Fig. 3. Sub-pixel motion estimation

\subsection{Quantization and DCT Optimization}

The weighting quantization method of H.264 has been introduced from high profile. Different quantization steps will be applied for various coefficients and regions with a scalar quantizer. The quantization method of H.264 is combined with transformation and quantization, thus the design of weighting quantization 
differs from the prior quantization matrix. The weighting quantization $C_{i j}$ at position $(i, j)$ is introduced as:

$$
C_{i j}=D_{i j} \times \operatorname{LevelScale}(Q P \% 6, i, j)>>\left(15+\text { floor }\left(\frac{Q P}{6}\right),\right.
$$

where LevelScale $(Q P \% 6, i, j)=\left(\frac{M F_{i j} \times 16}{\text { Scale }_{i j}}\right)$ in which $M F_{i j}$ denotes the multiplication factor (see Table112]), Scale $e_{i j}$ represents the value of the corresponding scaling table (illustrated in below), "\%" denotes the modular operation and $>>$ indicates a binary shift right operation.

The de-quantization factor $D_{i j}$ is computed as:

$$
D_{i j}=C_{i j} \times \text { DeLevelScale }(Q P \% 6, i, j)<<(Q P / 6-4),
$$

where DeLevelScale $(Q P \% 6, i, j)=V_{i j} \times S_{c a l e}{ }_{i j}$ in which $V_{i j}$ is defined in Table 2 for $0 \leq Q P \leq 5$, and $<<$ denotes the binary shift left operation. We can observe from the quantization function that after introducing quantization table, both forward and inverse quantization between $M F$ and $V_{i j}$ are scaled by a factor of Scale $_{i j}$. From our simulation results, after weighting quantization is applied, the video quality (PSNR) will be increased and the coding speed is also improved.

Table 1. Multiplication factor MF 12

\begin{tabular}{cccc}
\hline QP & $\begin{array}{c}\text { Positions } \\
(0,0),(2,0),(2,2),(0,2)\end{array}$ & $\begin{array}{c}\text { Positions } \\
(1,1),(1,3),(3,1),(3,3)\end{array}$ & Other positions \\
\hline 0 & 13107 & 5243 & 8066 \\
1 & 11916 & 4660 & 7490 \\
2 & 10082 & 4194 & 6554 \\
3 & 9362 & 3647 & 5825 \\
4 & 8192 & 3355 & 5243 \\
5 & 7282 & 2893 & 4559 \\
\hline
\end{tabular}

Table 2. Scaling factor $\mathrm{V}[12$

\begin{tabular}{cccc}
\hline QP & Positions & Positions & Other positions \\
& $(0,0),(2,0),(2,2),(0,2)$ & $(1,1),(1,3),(3,1),(3,3)$ & \\
\hline 0 & 10 & 16 & 13 \\
1 & 11 & 18 & 14 \\
2 & 13 & 20 & 16 \\
3 & 14 & 23 & 18 \\
4 & 16 & 25 & 20 \\
5 & 18 & 29 & 23 \\
\hline
\end{tabular}


Eight scaling tables are defined in below: including intra luma $8 \times 8$, intra chroma $\mathrm{U} 4 \times 4$, intra chroma $\mathrm{V} 4 \times 4$, inter luma $4 \times 4$, inter chroma $\mathrm{U} 4 \times 4$, inter chroma $\mathrm{V} 4 \times 4$, intra luma $8 \times 8$ and inter luma $8 \times 8$. The coefficients of the above mentioned equations are suggested by JVT [13].

$$
\begin{aligned}
\text { Intra } 4 \times 4 \_L u m a & =\text { Intra } 4 \times \text { 4_ChromaU } \\
& =\text { Intra } 4 \times 4 \_C h r o m a V
\end{aligned}=\left[\begin{array}{cccc}
0 & 12 & 19 & 26 \\
12 & 19 & 26 & 31 \\
19 & 26 & 31 & 35 \\
26 & 31 & 35 & 39
\end{array}\right],
$$

Inter $4 \times 4 \_L u m a=$ Inter $4 \times 4 \_C h r o m a U$

$$
=\text { Inter } 4 \times 4 \text { ChromaV }=\left[\begin{array}{cccc}
0 & 13 & 18 & 21 \\
13 & 18 & 21 & 24 \\
18 & 21 & 24 & 27 \\
21 & 24 & 27 & 30
\end{array}\right] \text {, }
$$

$$
\text { Intra8 } \times \text { 8_Luma }=\left[\begin{array}{cccccccc}
0 & 10 & 13 & 16 & 19 & 24 & 26 & 28 \\
10 & 12 & 16 & 19 & 24 & 26 & 28 & 31 \\
13 & 16 & 19 & 24 & 26 & 28 & 31 & 33 \\
16 & 19 & 24 & 26 & 28 & 31 & 33 & 35 \\
19 & 24 & 26 & 28 & 31 & 33 & 35 & 37 \\
24 & 26 & 28 & 31 & 33 & 35 & 37 & 39 \\
26 & 28 & 31 & 33 & 35 & 37 & 39 & 42 \\
28 & 31 & 33 & 35 & 37 & 39 & 42 & 44
\end{array}\right],
$$

$$
\text { Inter } 8 \times 8 \_ \text {_uma }=\left[\begin{array}{rrrrrrrr}
0 & 12 & 14 & 16 & 18 & 19 & 21 & 22 \\
12 & 13 & 16 & 18 & 19 & 21 & 22 & 24 \\
14 & 16 & 18 & 19 & 21 & 22 & 24 & 25 \\
16 & 18 & 19 & 21 & 22 & 24 & 25 & 27 \\
18 & 19 & 21 & 22 & 24 & 25 & 27 & 28 \\
19 & 21 & 22 & 24 & 25 & 27 & 28 & 30 \\
21 & 22 & 24 & 25 & 27 & 28 & 30 & 31 \\
22 & 24 & 25 & 27 & 28 & 30 & 31 & 33
\end{array}\right],
$$

$$
Y=C_{f} \times C_{f}^{T} \otimes E_{f}=\left[\begin{array}{cccc}
1 & 1 & 1 & 1 \\
2 & 1 & -1 & -2 \\
1 & -1 & -1 & 1 \\
1 & -2 & 2 & -1
\end{array}\right] \times\left[\begin{array}{cccc}
1 & 2 & 1 & 1 \\
1 & 1 & -1 & -2 \\
1 & -1 & -1 & 2 \\
1 & -2 & 1 & -1
\end{array}\right] \otimes\left[\begin{array}{cccc}
a^{2} & \frac{a b}{2} & a^{2} & \frac{a b}{2} \\
\frac{a b}{2} & \frac{b^{2}}{4} & \frac{a b}{2} & \frac{b^{2}}{4} \\
a^{2} & \frac{a b}{2} & a^{2} & \frac{a b}{2} \\
\frac{a b}{2} & \frac{b^{2}}{4} & \frac{a b}{2} & \frac{b^{2}}{4}
\end{array}\right]
$$


To simplify the implementation of the DCT transform and reduce multiplications by half in the transform of $C X C^{T}$ which could result in loss of accuracy using integer arithmetics on the DSP platform. The final forward transform is reformed as Eq. (7). We adopt a low-complexity transform proposed by Henrique S.Malvar [14, and lead to a new set of coefficients, which is shown in Fig. 4. There only consists of shift operations for FDCT and IDCT functions, which can benefit for the assembly code re-arrangement.
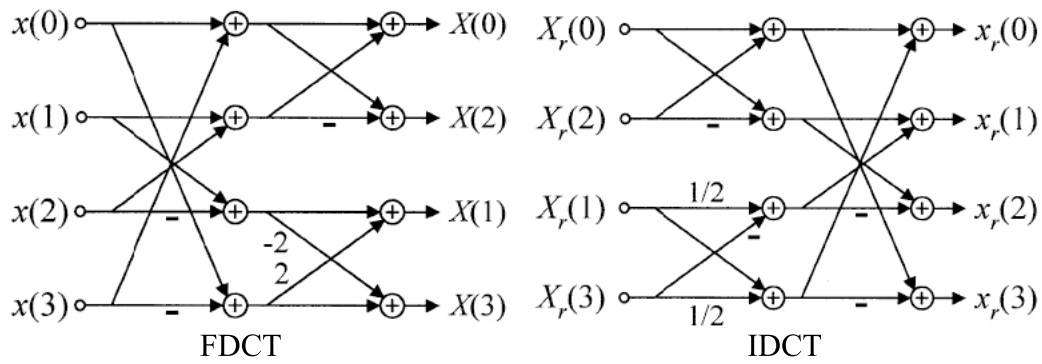

Fig. 4. Fast implementation of the H.264 direct transform [14]

\section{Experimental Results}

To evaluate the effectiveness of the proposed optimization algorithm, the optimized H.264 video encoder was implemented on the DM642 DSP platform. We set up a TI DM642EVM development environment including a target board, Code Composer Studio (CCS) profile tools, an input device (camera) and an output device (monitor) as shown in Fig. 5. Note that the rate control algorithm was not changed. Furthermore, system level optimization skills were also studied according to TI technical documents 151617181920]. The x264 encoder was

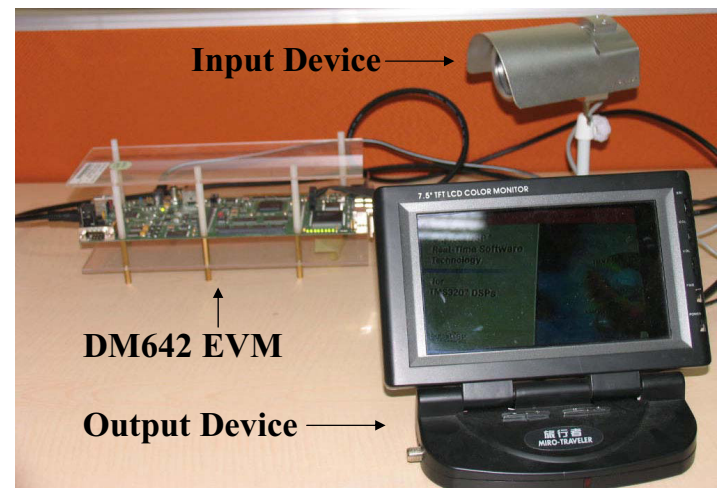

Fig. 5. Loopback system peripheral 


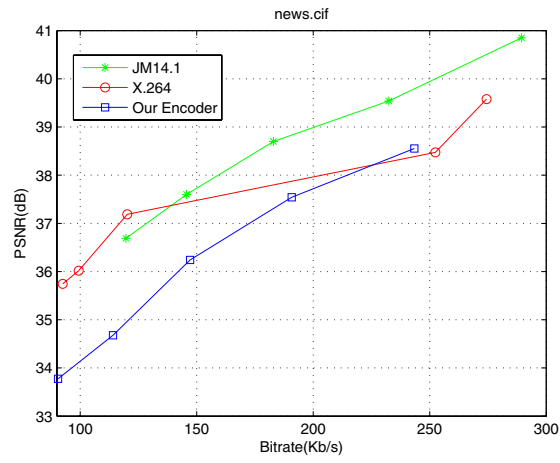

(a) News

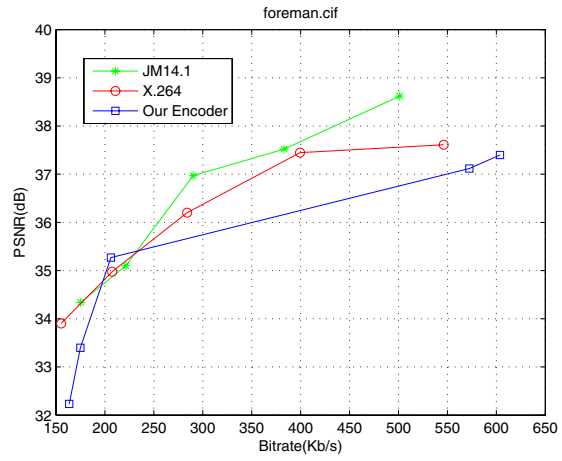

(b) Foreman

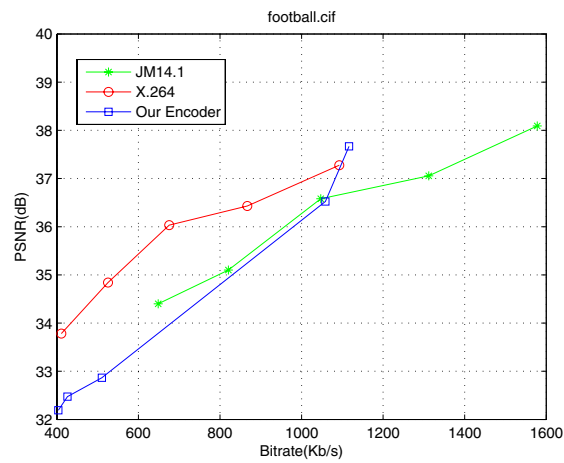

(c) Football

Fig. 6. The RD-curves of three codecs tested on three video clips: (a) News, (b) Foreman, and (c) Football

optimized and transplanted to DM642, and achieved the coding speed of 22.6fps for VGA $(640 \mathrm{x} 480)$ size video. The performance indices bitrate (Kbs), PSNR $(\mathrm{db})$ and FPS of each optimization approach has been measured on three benchmark video clips: news, football and foreman. Table 3 reveals and compares the progress of all optimization steps, including (a) direct porting of baseline x264 encoder, (b) quantization and DCT optimization, (c) sub-pixel motion estimation optimization, and (d) 2-D fast mode decision optimization. The overall performances with DSP implementation and optimization are listed in the most right column in Table 3 .

In order to evaluate the PSNR and bitrate of the optimized encoder, we compare our encoder with JM14.1 and x264 on Intel CPU T2250 @ $1.73 \mathrm{GHz}$ platform with 1GB DDR RAM. Figures 6(a), (b) and (c) demonstrate the RDcurves of these three codecs with different QP values 26, 28, 30, 32 and 34 for CIF video clips News, Foreman and Football, respectively. Although our encoder results in less PSNR (all less than $2 \mathrm{db}$ ), the proposed encoding speed is increased up to 30-50 fps which is faster than the orignal X.264. 
Table 3. The performance indices bitrate (Kbs), PSNR (db) and FPS of the implemented codec on DM642 with different optimization step: (a) direct porting of baseline x264 encoder, (b) quantization and DCT optimization, (c) sub-pixel motion estimation optimization, and (d) 2-D fast mode decision optimization, and overall results

\begin{tabular}{|c|c|c|c|c|c|c|}
\hline \multirow[b]{2}{*}{$\begin{array}{l}\text { Video } \\
\text { clips }\end{array}$} & & \multicolumn{5}{|c|}{ Optimization Step } \\
\hline & & (a) & $(a)+(b)$ & $\begin{array}{c}(\mathrm{a})+(\mathrm{b}) \\
+(\mathrm{c})\end{array}$ & $\begin{array}{r}(\mathrm{a})+(\mathrm{b}) \\
+(\mathrm{c})+(\mathrm{d}) \\
\end{array}$ & $\begin{array}{l}\text { Overall } \\
\text { result }\end{array}$ \\
\hline \multirow{3}{*}{ News } & BitRate & 307.42 & 294.877 & 254.825 & 203.305 & 252.46 \\
\hline & PSNR & 40.823 & 40.274 & 39.647 & 38.68 & 38.533 \\
\hline & FPS & 15.30 & 15.59 & 29.22 & 32.94 & 42.50 \\
\hline \multirow{3}{*}{ Football } & BitRate & 1971.71 & 1983.12 & 1454.13 & 1132.42 & 1058.47 \\
\hline & PSNR & 40.589 & 41.764 & 38.324 & 38.208 & 37.669 \\
\hline & FPS & 7.09 & 7.61 & 15.97 & 21.45 & 31.95 \\
\hline \multirow{3}{*}{ Foreman } & BitRate & 1003.43 & 1014.67 & 908.95 & 869.77 & 872.76 \\
\hline & PSNR & 40.103 & 40.928 & 38.427 & 37.692 & 37.399 \\
\hline & FPS & 10.49 & 11.77 & 22.69 & 29.35 & 36.64 \\
\hline
\end{tabular}

\section{Conclusion}

In this paper, we optimize the H.264/AVC baseline video encoder based on x264 codec and implement the optimized codec on TI DM642 DSP platform. A series of optimization methods have been proposed, including 2-D fast mode decision, sub-pixel optimization for motion estimation, and weighted matrix quantization. Both code level and system level optimization principals are adopted. Experimental results reveal that the optimized H.264 video encoder retains satisfactory quality with very low degradation. The implemented codec can achieve the coding speed of 22.6fps and more than 40fps for VGA $(640 \times 480)$ and CIF $(352 \times 288)$ resolution, respectively. The proposed H.264 codec can be employed in many real-time applications. Compared to the special purpose IC solution of H.264 codec, the embedded DSP system has advantages of high flexibility and re-configuration.

\section{References}

1. Goto, K., Hatabu, A., Nishizuka, H., Matsunaga, K., Nakamura, R., Mochizuki, Y., Miyazaki, T.: H.264 video encoder implementation on a low-power DSP with low and stable computational complexity. In: IEEE Workshop on Signal Processing Systems Design and Implementation, SIPS 2006, pp. 101-106 (2006)

2. Chen, T.-C., Chen, Y.-H., Tsai, S.-F., Chien, S.-Y., Chen, L.-G.: Fast algorithm and architecture design of low-power integer motion estimation for H.264/AVC. IEEE Transactions on Circuits and Systems for Video Technology 17(5), 568-577 (2007) 
3. Zhuo, L., Wang, Q., Feng, D.-D., Shen, L.: Optimization and implementation of H.264 encoder on DSP platform. In: IEEE International Conference on Multimedia and Expo., 232-235 (2007)

4. Li, Z., Xing, Q., Zhu, X.: H.264 video encoder implementation and optimization based on DM642 DSP. In: IEEE International Conference on Networking, Sensing and Control, ICNSC 2008, pp. 891-894 (2008)

5. Wei, Z., Cai, C.: Realization and optimization of DSP based H.264 encoder. In: Proceedings. 2006 IEEE International Symposium on Circuits and Systems, ISCAS 2006, p. 4 (2006)

6. Wang, H.-J., Hou, Y.-Y., Li, H.: H.264/AVC video encoder algorithm optimization based on TI TMS320DM642. In: Third International Conference on Intelligent Information Hiding and Multimedia Signal Processing, IIHMSP 2007, vol. 1 (2007)

7. Werda, I., Chaouch, H., Samet, A., Ayed, M.A.B., Masmoudi, N., Akbal, E., Ergen, B., Muljadi, H., Takeda, H., Ando, K., et al.: Optimal DSP-based motion estimation tools implementation for H.264/AVC baseline encoder. IJCSNS 7(5), 141 (2007)

8. Wiegand, T., Sullivan, G.J., Bjntegaard, G., Luthra, A.: Overview of the H.264/AVC video coding standard. IEEE Transactions on Circuits and Systems for Video Technology 13(7), 560-576 (2003)

9. Texas Instrument. TMS320C64x DSP Video Port/VCXO Interpolated Control Port (2006)

10. LFree Software Foundation. GNU operating system, http://www.gnu.org/

11. Chang, C.-Y., Pan, C.-H., Chen, H.: Fast mode decision for P-frames in H. 264. In: Picture Coding Symposium (PCS) (2004)

12. Richardson, I.E.G.: H. 264 and MPEG-4 video compression. Wiley, Chichester (2003)

13. J.V. Team. Draft ITU-T recommendaation and final draft international standard of joint video specification (March 2003)

14. Malvar, H., Hallapuro, A., Karczewicz, M., Kerofsky, L.: Low-complexity transform and quantization in H.264/AVC. IEEE Transactions on Circuits and Systems for Video Technology 13(7), 598-603 (2003)

15. Texas Instrument. TMS320C6000 Assembly Language Tools v6.0 Beta (2005)

16. Texas Instrument. Code Composer Studio User's Guide (2000)

17. Texas Instrument. TMS320C6000 DSP/BIOS User's Guide (2000)

18. Texas Instrument. TMS320C64x/C64x+ DSP CPU and instruction set reference guide

19. Texas Instrument. Video Encoding Optimization on TMS320DM64x/C64x (2004)

20. Texas Instrument. TMS320C64x DSP Two-Level Internsl Memory Rfference Guide (2006) 